\section{Use of a second transcatheter Rashkind arterial duct occluder for persistent flow after implantation of the first device: indications and results}

\author{
I C Huggon, A H Tabatabaei, S A Qureshi, E J Baker, M Tynan
}

\begin{abstract}
Objective-To assess the efficacy, feasibility, and most appropriate timing of the implantation of a second Rashkind arterial duct occluder because of persistent flow after the first device.

Design-A prospective serial Doppler study of patients after the insertion of a Rashkind arterial duct occluder including a subgroup in whom a second device was implanted.
\end{abstract}

Setting-A tertiary referral centre for congenital heart disease.

Patients-144 patients aged 7 months to 67 years (median 3.38 years) who underwent transcatheter occlusion of patent arterial duct, 20 of whom had attempted implantation of a second device.

Interventions-Implantation of a second device alongside the first was attempted in 20 of the patients with persistent residual flow.

Main outcome measures-Successful implantation of a second device, the incidence of complications, and the achievement of complete occlusion on follow up Doppler echocardiography. The time to complete occlusion in the whole group and factors predictive of persistent leak were also analysed.

Result-Second devices were successfully implanted in 19 of 20 first attempts and in the remaining patient at the second attempt. Complete occlusion was found in 19 patients at a mean follow up of eight months. The complications included fracture of a guidewire requiring femoral arteriotomy for its removal in one patient and embolisation of a device in another. With a single device, persistence of residual flow six months after implantation and malposition of the device on the aortogram after implantation were predictive of continuing residual patency. Paediatric Cardiology, Guy's Hospital, London

I C Huggon

A H Tabatabaei

S A Qureshi

E J Baker

M Tynan

Correspondence to Dr S A Qureshi, Paediatric Cardiology, 11 th Floo Guy's Tower, Guy's Hospital, St Thomas Street, London SE1 9RT.

Accepted for publication 18 January 1993.

Conclusions-Implantation of a second device is safe, feasible, and effective and persists beyond six months, or if position of the first device causes complications such as haemolysis.

(Br Heart f 1993;69:544-550)
Department of thoracotomy scar, reduced stay in hospital $\vec{C}$ and patient and parent preference. ${ }^{12}$ 을 Currently, the only commercially available $\frac{\bar{\omega}}{\vec{\sigma}}$ system is the Rashkind double umbrella $\stackrel{\varnothing}{\varnothing}$ device that can be delivered through the femoral venous route and is applicable to $\vec{\circ}$ most patients with a patent arterial duct beyond infancy and to some infants as small $\vec{\omega}$ as $6 \mathrm{Kg}$. A disadvantage of this technique is $\frac{\mathscr{O}}{\mathrm{T}}$ that the incidence of residual leak after implantation of a single device remains higher 8 than that generally reported for surgical liga- os tion of the arterial duct. ${ }^{2-4}$ This shortcoming $A$ may be overcome by implantation of a second 0 Rashkind device alongside the first in patients with a persistent residual leak. ${ }^{5-7}$

The purposes of this paper are to define the time to complete occlusion after implan- $\vec{\theta}$ tation of a single device, to find the most $\&$ appropriate time for implantation of a second device in those patients with a persistent leak, and to report the results of attempted implantation of a second device and make some general recomendations about its application.

\section{Patients and methods}

\section{PATIENTS}

In our hospital 144 patients have had a first Rashkind occlusion device successfully placed in the patent arterial duct since May 1986. Ninety nine patients had the $12 \mathrm{~mm}$ device and 45 the $17 \mathrm{~mm}$. Each patient had serial $\frac{5}{3}$ Doppler echocardiographic studies at intervals to determine the patency of the duct.

The ages at the time of the implantation ranged from seven months to 67 years (median 3.38 years) and the weight from 6.15 to $\frac{7}{0}$ $79.6 \mathrm{~kg}$ (median $14.28 \mathrm{~kg}$ ). In one patient surgical removal of the device combined with $\sigma$ ligation of the duct was necessary because of $N$ severe haemolysis. ${ }^{8}$ Of the remaining $143 \mathrm{~N}$ patients, $68(47 \%)$ had complete occlusion by the day after implantation and a further $30_{\circ}^{\circ}$ had developed occlusion during a follow up of one to 43 months. Thus at the latest follow $\stackrel{?}{?}$ up, 98 patients $(68.5 \%)$ had confirmed com- Tㅜㅇ plete occlusion on Doppler echocardiography after a single device, and 45 patients $(31.5 \%) \stackrel{?}{\stackrel{?}{?}}$ had residual flow. Twenty of these $45 \AA$ patients have had second devices implanted and four are awaiting second devices. The 8 remaining 21 patients have an echocardiographic follow up of less than six months, either because of a recently implanted device (15 patients) or deficient follow up (six patients). Only one patient with haemolysis, already mentioned, has had surgical treatment for a residual leak.
Transcatheter occlusion of the patent arterial duct offers considerable advantages over surgical ligation. These include avoidance of a 
The procedure and outcome for the 20 patients in whom there have been 21 attempts at implantation of a second device are described. All patients underwent cross sectional echocardiography with colour flow mapping and pulsed and continuous wave Doppler studies before implantation of the second device. In these patients, the interval between implantation of the first and the second device ranged from one to 43 months (mean 16.1 months), the age at insertion of the second device ranged from 0.92 to 10.2 years (median 3.3 years) and the weight from 6.7 to $28.9 \mathrm{~kg}$ (median $14.35 \mathrm{~kg}$ ). The patients selected were those with residual leaks in whom the first device had been implanted at least four months previously. During our early experience, two patients had second devices implanted one month (a 10 month old child with pulmonary hypertension, and atrioventricular septal defect, and significant residual ductal flow) and three months after the first procedure. Of 21 attempts, two were less than four months after implantation of the first device, four were between four and six months, three between six months and a year, eight between one and two years, and four more than two years. In the past two years, in 11 patients who had a second device implanted, the interval between the implantation of the two devices was more than 11 months.

Before implantation of the second device, all 20 patients had continuous flow on Doppler echocardiography of the residual duct. Thirteen of these had continuous murmurs, and seven had systolic murmurs.

\section{TECHNIQUE}

The patients were prepared for cardiac catheterisation in the usual way. Informed consent was obtained from the parents. Five of the patients had general anaesthesia and endotracheal intubation for the procedure and the rest had local anaesthetic infiltration of the groins together with sedation with diazemuls and ketamine. The technique used was similar to that used for implantation of the first device. ${ }^{2}$ In six patients in whom the long sheath and dilator did not pass easily across the residual duct a complete arteriovenous guidewire circuit was established to facilitate this as has been described for small native ducts. ${ }^{9}$ The guidewire circuit was established by passing a snare from the femoral artery to grasp the tip of the guidewire in the aorta and carefully pulling the guidewire, shielded for most of its length by a catheter, out through the arterial sheath. In two cases dilation with catheters of increasing size was required as well as the guidewire circuit. Once the long sheath was satisfactorily positioned, the guidewire circuit was broken and the loaded delivery system was passed through the sheath. Positioning and release of the second device was as previously described for a first device ${ }^{2}$ except that the sheath was advanced slightly to come into contact with the device before release. This manoeuvre has been developed for use during implantation of both first and second devices to avoid snagging of the knuckle in the foam of the device during release and thus minimising the possibility of embolisation.

In 18 of the patients in whom the residual duct was less than $3.5 \mathrm{~mm}$ in diameter on the aortogram, the second device was of $12 \mathrm{~mm}$, whereas in two, $17 \mathrm{~mm}$ devices were used. Both of these two had also had $17 \mathrm{~mm}$ devices on the first occasion. An aortogram was taken before release of the device to confirm a satisfactory position of the proximal and distal prongs. A further aortogram was taken after release of the device.

\section{ASSESSMENT OF OCCLUSION}

On the day after the procedure, cross sectional echocardiography with colour flow mapping, pulsed wave, and continuous wave Doppler studies were performed with a Sonotron CFM 750 system (Vingmed). Complete occlusion on the Doppler study was defined as the absence of any residual flow after careful examination in a Cooperative or sedated patient. Patients with a residual leak on the first day after the procedure were scheduled for echocardiographic assessment at about six weeks and at three to six monthly intervals thereafter if the leak persisted.

The actuarial curve with $95 \%$ confidence intervals for residual flow was calculated from the Kaplan-Meier product limit analysis of the intervals ${ }^{10}$ to complete occlusion in all 144 patients. Patients whose duct was not occluded at the last follow up, or who had a second device implanted were entered as censored in the analysis. For patients whose ducts were completely occluded, the time of complete occlusion was considered to be the mid point between the time of echocardiographic assessment showing complete occlusion and the last time at which flow was still shown to be present.

To compare our results with those of others, the Kaplan-Meier analysis was repeated with the same assumptions made by Musewe et $a l^{4}$ and Hosking et al. ${ }^{3}$ They assumed that in every case, complete occlusion occurred when flow was shown to be absent on the Doppler study. We did not, however, exclude patients who later had second devices implanted, as did Hosking et al, but instead included them as "censored" in the analysis at the time that the second device was implanted. We cannot see any justification for excluding this group of patients selected on the basis that their ducts were not expected to close and believe that their exclusion introduces an avoidable bias. A further curve was constructed in which we assumed that in the patients who developed complete occlusion, the occlusion occurred immediately after the last Doppler study that showed residual flow.

\section{DEFINITION OF MALPOSITION OF A DEVICE}

Malposition of the duct occlusion device was defined as important discrepancy of the position of the hinge point and the narrowest point of the arterial duct. The position of the 
distal and proximal prongs of the device in relation to the duct ampulla and pulmonary artery were also considered in defining malposition.

\section{Results}

RESULTS OF IMPLANTATION OF SECOND

DEVICES

Implantation

In 19 of the 20 patients, the second Rashkind device was satisfactorily positioned and released alongside the first device at the first attempt. In one patient the second device could not be implanted as the residual duct was very small, only allowing the passage of 0.025 inch guidewire but not a 6 French catheter. This patient had a continuous murmur. At a further attempt 43 months after implantation of the first device, a $12 \mathrm{~mm}$ device was implanted with the use of a complete arteriovenous guidewire circuit and this resulted in complete occlusion. Thus all 20 patients in whom a second device was attempted have two devices in position.

The first device was of $12 \mathrm{~mm}$ diameter in 11 patients and $17 \mathrm{~mm}$ in nine patients. The second device was of $12 \mathrm{~mm}$ diameter in all except two patients who received the $17 \mathrm{~mm}$ device.

\section{Complications}

Two important complications occurred in association with implantation of the second device. In one patient, the guidewire, which had been snared to establish a complete arteriovenous circuit, broke as it was being pulled into the arterial sheath and a fragment lodged in the femoral artery. After implantation of the second Rashkind device, the guidewire fragment was removed by a femoral arteriotomy the next day. In another patient, the 12 $\mathrm{mm}$ device embolised to the right pulmonary artery. It was removed with a retrieval catheter and another $12 \mathrm{~mm}$ device was implanted at the same procedure.

During the follow up none of the patients have developed endarteritis or stenosis of the origin of the left pulmonary artery associated with the devices.

\section{Occlusion rate after second device}

The complete occlusion rate after the second device was $6 / 20(30 \%)$, when assessed by angiography immediately at the end of the procedure. By the next day this had increased to $15 / 20(75 \%)$ when assessed by Doppler echocardiography. Of the remaining five patients, only one $(5 \%)$ patient has a persistent leak after 29 months of follow up. In the other four patients, complete occlusion occurred between one day and seven months in one and between one month and three months in two. The fourth patient had a residual leak at seven months but did not have repeat Doppler study until two years after implantation of the second device, when complete occlusion was confirmed.

Thus at the latest follow up of between one and 29 months (mean eight months), 19
(95\%) of the arterial ducts with two devices in place were completely occluded on Doppler echocardiography and colour flow mapping. Overall, $117(81 \cdot 1 \%)$ of the 143 patients with one or two devices in place had complete occlusion.

RESULTS OF IMPLANTATION OF FIRST DEVICES Time to occlusion after a first device

Because residual flow or complete occlusion $c$ can only be assessed accurately by Doppler $\Rightarrow$ echocardiography at intervals dictated by the $\stackrel{?}{?}$ patients' attendance for follow up, only the? interval during which the occlusion occurred $\frac{\overline{\bar{C}}}{\bar{\rho}}$ rather than the exact time at which it $\overrightarrow{\widetilde{\alpha}}$ occurred can be determined.

Figure 1 shows the results of the KaplanMeier analysis of the 144 patients after their $\vec{\circ}$ first device, based on the assumption that the time of complete occlusion was the midpoint ${ }_{\sigma}^{\omega}$ of the last time that residual flow was shown? and the first time this flow was shown to be absent. The curve shows that $84 \%$ of the ducts had residual flow (16\% complete occlu-ir sion) on angiography immediately after $\Rightarrow$ implantation of a device, $52 \%$ had residualo flow (48\% complete occlusion) on the next-

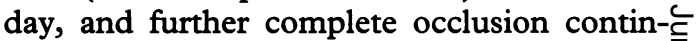
ued to occur so that the complete occlusion $\bar{\sigma}$ rate was shown to be $70 \%$ by seven months $\vec{\circ}$ and $77 \%$ by one year. The rest of the patients $\Theta$ were assumed to have residual flow unless proved otherwise.

Of the 98 patients with completely occlud-ed arterial ducts after the first device 21 had@ complete occlusion confirmed more than six $\frac{\mathscr{Q}}{\mathbb{Q}}$ months after implantation. This was because $\underset{\vec{A}}{\overrightarrow{0}}$ of the timing of the Doppler examinations 3 rather than actual occlusion occurring at that time. Only one of these patients actually hadọ proved residual flow after six months (this became completely occluded between seven months and a year). The remaining $20 \frac{-}{-}$ patients had not had Doppler assessment 3 . between six months and the time at which complete occlusion was confirmed.

FACTORS INFLUENCING RESULTS 132 of the 144 patients, for whom the original angiograms were available, showed that $13(10 \%)$ patients had apparent malposition ${ }^{N}$ of the device, with one or more distal prongs seeming to stent open the duct. Four of these ducts, however, were completely occluded을 despite the malposition, all of them by the day after implantation. None of the remain-ing nine patients $\left(69 \%\right.$ of those with malposi- $-\frac{0}{0}$ tion) developed complete occlusion without further intervention. One of these was in the $\frac{?}{\mathbb{C}}$ patient who required surgery for severe $\varrho$ haemolysis associated with the residual leake eight days after implantation; five have hado second devices for residual flow and the? remaining three still have a residual leak buto have a short mean follow up of five months. (range 1.5 to 6.5 months). One of the patients with malposition is the only patient with a residual leak after implantation of a second device. Before the insertion of the first 


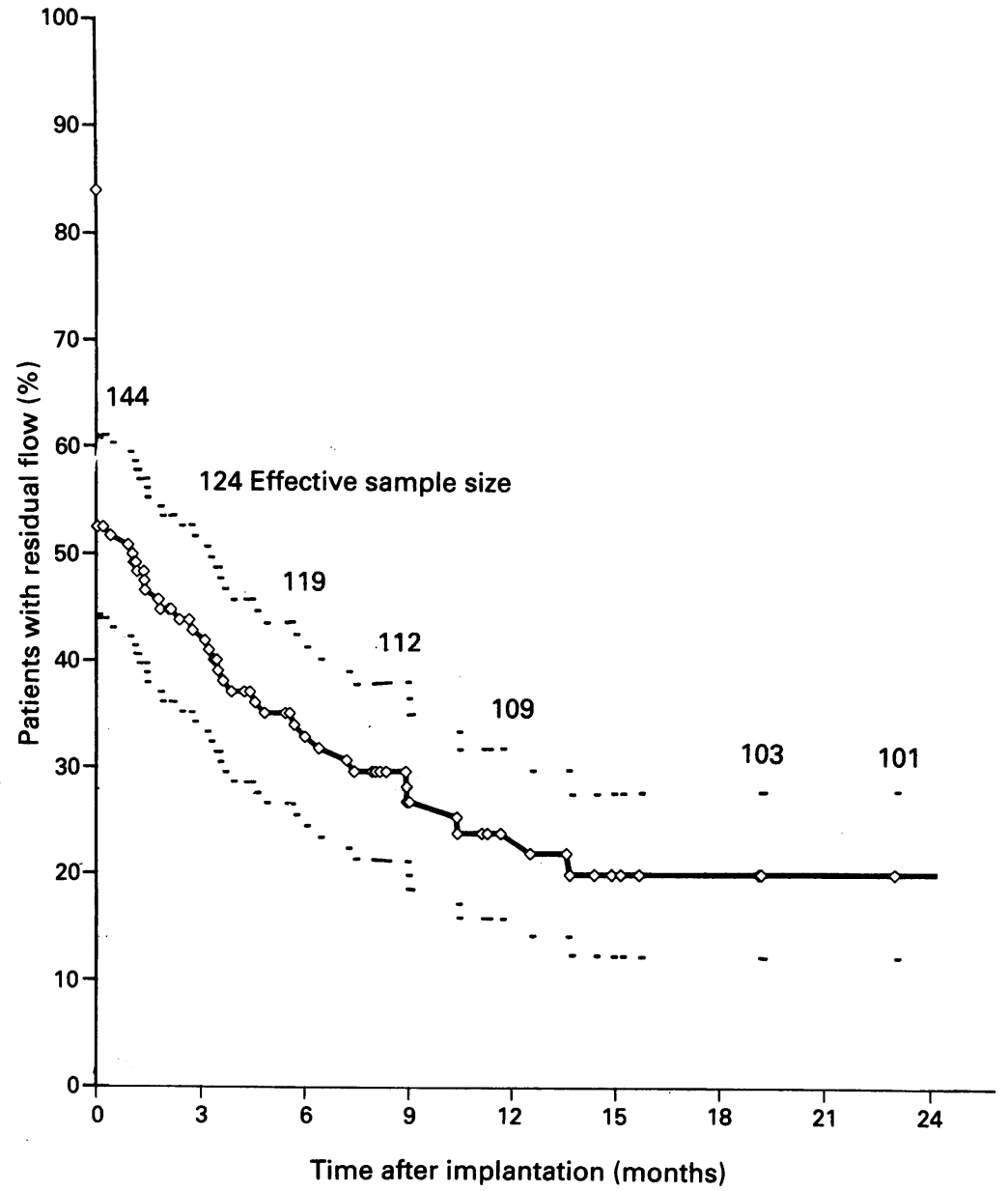

Figure 1 Diegram based on a isplan-Meier analysis afieximplantation of a first device. The time of occlusion is assumed to be the midpoint of the inverval between the last time that flow was shown and the first time that it was shown to be absent. Dives not yet occluded, those in which a second device was implanted, and one that was ligated were entered into the analysis as values censored at the time that flow was last shown. $95 \%$ CIs are given. also advisable to operate upon all children and young adults who have an open ductus, believing that this prophylactic procedure is of real value in warding off cardiovascular complications in later years." 12 More than 30 years later Kirklin and Barrat-Boyes recommended that "the presence of persisting patency of the PDA is an indication for its surgical closure."13 It is also thought that "subacute bacterial endocarditis occurs mainly as a complication of a small PDA and less often with a moderate-sized ductus."13 Surgery has become the accepted method of treatment over the years and is associated with mortality approaching $0 \% .^{13}$ The technique used has been either ligation or division of the duct. In the modern era, ligation has been preferred to division because of its greater safety. The presumption was that ligation or division resulted in complete closure of the duct and thus removed the risks of endocarditis. The view held over the years, that closure of the arterial duct is always indicated, has precluded up to date studies of the natural history. More sensitive diagnostic techniques, improved antibiotic treatment, and recognition of residual flow after apparently successful duct ligation all raise doubts as to whether this view remains valid. Alternatively if this view is accepted, and in clinical practice it has been, then any residual flow through the arterial duct will predispose the patient to risks of endocarditis.

In the past it has been argued that surgical treatment for a patent arterial duct was superior to the transcatheter techniques because it was believed that very few ducts remained patent after surgical ligation. ${ }^{13}$ This belief, however, has been shown to be misplaced because when patients who had had surgical ligation of the duct were assessed with Doppler colour flow mapping, an incidence of residual flow of $6 \%-23 \%$ was found..$^{414}$ This dampened the concern that the incidence of residual leak after the Rashkind device was too high. Whereas implantation of a second Rashkind device when flow persists after the first device overcomes the problem of a high incidence of residual leak, the same cannot be said of residual patency after surgical ligation. In our series we have succeeded in implanting an occluding device in only one out of four patients with residual patency of the duct after previous surgical ligation. With complete occlusion rates of $77 \%$ at one year after one device and $95 \%$ in those patients with a second device, occlusion rates comparable with or better than surgical ligation should be possible provided that implantation of a second device is attempted in all those patients with persisting residual leaks.

An important consideration for the implantation of second devices is the most appropriate timing. In view of the information now available about the time to complete occlusion, the only definitive indication for attempting implantation of a second device earlier than six months after the first is haemolysis, ${ }^{15}$ and a possible indication is a situation when there is difficulty in assessing

\begin{abstract}
the fact that in the presence of a patent arterial duct, even if bacterial endocarditis was successfully treated, the risk of further attacks remained. Furthermore he stated that "it is

In 1968 Maurice Campbell stated that patients with patent arterial ducts were heart failure. 11 In the era before antibiotics and surgery bacterial endocarditis carried a high mortality, but the advent of antibiotics
\end{abstract}


the pulmonary vascular resistance in patients with other associated intracardiac defects. Therefore it seems likely that a few patients early in our series, who had second devices implanted within six months of the first device, might have developed complete occlusion had they been left a little longer without a second device. It is our current policy for those patients with residual flow at six months or more after the first device to undergo a second procedure on a routine basis, although in practice they usually have their second procedure about a year after the first. With this policy no patient has developed complete occlusion while waiting for a second device.

Our approach may be at variance with other units because of different methods of using the Kaplan-Meier analysis. ${ }^{34}$ In a recent paper, Hosking et al argued that complete occlusion continued to occur well beyond the first year after implantation and imply that procedures for second devices should therefore be deferred. ${ }^{3}$ Their findings were based on a Kaplan-Meier analysis in which they assumed that complete occlusion actually occurred at the time when no residual flow could be shown on a Doppler study and did not take into account the fact that observations are made only intermittently. There are logistical and methodological problems in assessing the exact closure time. Residual flow or complete occlusion can only be assessed by Doppler echocardiography performed at intervals dictated by the unit's

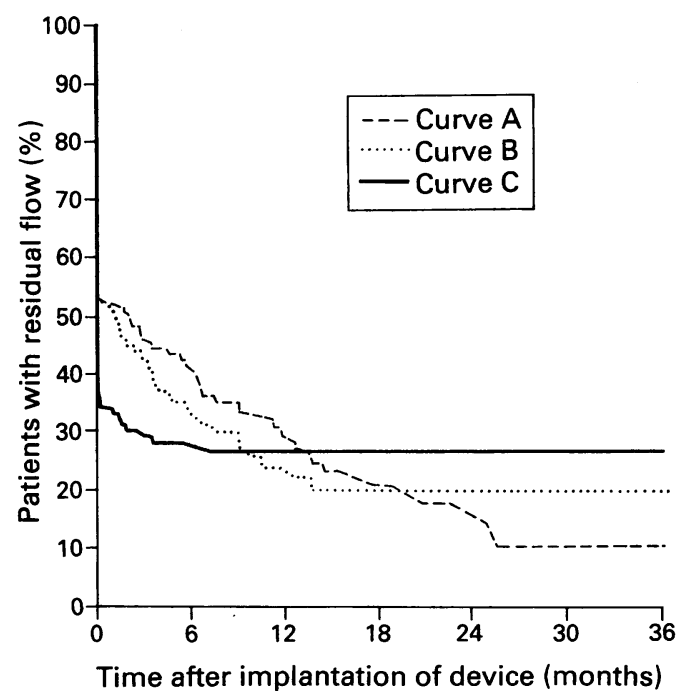

Figure 2 Kaplan-Meier analysis for residual flow analogous to fig 1 but showing the effect on the curve of three alternative assumptions about the time of the occurrence of complete occlusion in relation to the Doppler studies that last showed flow and that first showed flow to be absent. (A) Assumes that where complete occlusion occurred, the time of occlusion was immediately before the Doppler study that first showed no flow. This is similar to the analysis of Hosking et al. ${ }^{3}$ (B) Assumes that where complete occlusion occurred, the time of occlusion was
midway between the last Doppler investigation to show midway between the last Doppler investigation to show is the same as the curve in fig 1. (C) Assumes that where complete occlusion occurred, the time of occlusion was immediately after the last Doppler study that showed residual flow. The differing assumptions made resultant residual flow. The differing assumptions made resultant
curves that differ not only in their slope, but also in the residual flow rate at the longest follow up intervals. workload and the patients' attendance. Therefore only the interval during which occlusion occurred rather than the exact time at which it occurred can be determined. Figure 2 curve A shows an analysis of our own data, with the same assumptions as made by Hosking et al. This curve is very similar to the one in the paper of Hosking et $a l$, both in terms of the slope and the rates of residual flow. Figure 2 curve $C$ results when the opposite assumptions are made (that in patients with complete occlusion, residual flow ceased directly after the last Doppler examination showing residual flow). This curve shows a rapid decrease in the proportion of patients with residual flow in the early weeks after implantation and almost no further occlusion after six months. Curve $B$ in fig 2 represents the proportion of patients with residual flow, with the assumption that occlusion occurred at the mid point of the interval between the two Doppler studies (the last one that showed residual flow and the first one showing occlusion). A precise curve can only be derived if the intervals between Doppler observations are infinitely small. When the intervals between the Doppler studies are as great as six months to a year, we have shown that the curves can be considerably and variably skewed depending on the assumptions that have been used. Our own belief is that the true curve for residual flow (that which would be produced by very frequent observations of ductal patency) lies somewhere between curves B and C. We contend that in most cases when complete occlusion is shown late, this occurs because the patient had not had a Doppler examination until a long time after occlusion had actually occurred. We do not deny that late complete occlusion can and does occur, as in one of our patients, but we dispute the suggestion that there is good evidence that very late occlusion is a common occurrence making implantation of a second device unnecessary. To determine with certainty the true time of closure after implantation, much more frequent echocardiographic assessment of patients will be necessary.

Another controversy surrounds the importance of small amounts of residual flow. The principle rationale for closing both native and residual ducts is to avoid a continuing risk of $N_{\omega}^{N}$ infective endarteritis associated with the $\sigma^{2}$ residual flow. From historical studies, the risk 0 of endarteritis in clinically apparent arterial ducts is estimated to be between $0.45 \%$ and $1 \%$ for each year of the patient's life, with the risk increasing with the patient's age. ${ }^{11}$ Improvements in diagnostic sensitivity, in particular Doppler colour flow mapping, have led to the discovery of ductal flow in the absence of the classical clinical signs as a coincidental finding in otherwise normal children and adults ${ }^{16}$ and in patients who have previously had apparently successful duct ligation. ${ }^{14}$ The risk of endarteritis for patients in these groups is uncertain. Endarteritis has not, as far as we are aware, been reported in patients in any of these groups. The only case 
of endarteritis associated with residual flow late after transcatheter occlusion had classical clinical signs of patent arterial duct. ${ }^{17}$ The fact that a significant proportion (22\%) of cases of endocarditis occurs without an identified predisposing cardiac abnormality ${ }^{18}$ means that such previously unrecognised abnormalities may be a predisposing factor. ${ }^{16}$ Also, in the absence of cardiac clinical signs, endarteritis from such a silent duct might have been treated as a bacteraemia of unknown cause. The presence of a foreign body such as an umbrella device at the site of residual flow in the duct may affect susceptibility to infection. Hosking $e t a l^{3}$ are in agreement with our own view, that, when an occlusion device has been implanted in the duct and residual flow is present, then antibiotic prophylaxis should be given for dental and other procedures for the duration of the residual flow. A recent report from The Netherlands calls into question the value of antibiotic prophylaxis, specifically in relation to valvar heart disease. ${ }^{19}$ This report confirms previous findings that only a minority $(12.7 \%)$ of cases of endocarditis result from dental or similar procedures, ${ }^{18}$ and indicates that recommended antibiotic prophylaxis is only actually given for a minority (25\%) of procedures for which it is indicated. Even when given appropriately, it has a protective efficacy of only $49 \%$ at the highest estimate. ${ }^{18}$ There is no reason to suppose that antibiotic prophylaxis is any more effective than this in patent arterial duct. We consider it illogical to advocate antibiotic prophylaxis for residual leaks without also offering treatment to abolish the leak when this is readily available. The high complete occlusion rate after a second device, the relative ease of implantation, and doubts about the efficacy of antibiotic prophylaxis have encouraged us in the view that when the chance of residual flow resolving spontaneously becomes low, the patient should be offered implantation of a second device to fulfil the aims of the first procedure. Firm evidence of the importance of small residual leaks is still lacking, but until better information becomes available as to what constitutes small leaks we consider it prudent to treat them similarly to larger leaks, which carry a proved risk of endarteritis ${ }^{17}$ rather than to consider them trivial.

It has been recommended by others that implantation of second devices be restricted to patients with continuous murmurs. This was on the basis that the residual duct could not be crossed in the one patient without a continuous murmur in whom the procedure was attempted and that flow not associated with continuous murmurs was likely to resolve spontaneously. ${ }^{37} \mathrm{We}$ believe that this recommendation may be misleading. Our own experience shows that implantation of a second device is feasible in patients with residual flow even in the absence of continuous murmurs. Indeed, we have found the presence or absence of a continuous murmur unhelpful in assessment. One of our patients, in whom a second device was not implanted at the first attempt and who had one of the smallest residual ducts, had a classical continuous murmur. The technique for implanting a second device is not as difficult as that for a first device as the first device provides a fixed landmark for reference. Because the residual duct is usually small, dilatation with catheters of increasing size may be required first, and in cases of difficulty the establishment of an arteriovenous guidewire circuit has been helpful. Once the circuit has been established, the operator has much greater control in manipulation of the dilator and sheath through a tight or unusually directed residual duct. The positioning of the second device is generally easier because of the presence of the first device.

Theoretically, malposition of the device may be expected to predispose to a residual leak after the first device. In our experience, however, complete occlusion can still occur within 24 hours in some patients with a badly positioned device. In other patients, with a persistent leak associated with malposition, a second device is needed and this should be done earlier than six months only if there is a complication such as haemolysis.

The implantation of a second Rashkind device is feasible, when flow persists after the first device. It has a high rate of success in completely occluding the duct and a low incidence of complications. Future transcatheter duct occlusion devices should be designed to fit ducts of a wider variety of shapes and sizes to avoid the possibility of malposition giving rise to residual leak. Despite some deficiencies, however, the currently available device performs satisfactorily in most patients with patent arterial duct and is the preferred treatment in adults, older children, and large infants. Whether small residual leaks are associated with risks of endocarditis needs further evaluation. Implantation of a second device is a satisfactory means of abolishing persistent residual flow and needs to be considered if flow persists beyond six months.

We are grateful to $\mathrm{Mr} \mathrm{N}$ Smeeton for statistical advice.

1 Latson LA, Hofschire PJ, Kugler JD, Cheatman JP, Gumbiner CH, Danford DA. Transcatheter closure of patent ductus arteriosus in pediatric patients. $f$ Pediat 1989;115:549-53.

2 Rashkind WJ, Mullins CE, Hellenbrand WE, Tait MA. Nonsurgical closure of patent ductus arteriosus: clinical application of the Rashkind PDA occluder system. Circulation 1987;75:583-92.

3 Hosking MCK, Benson LN, Musewe N, Dyke JD, Freedom RM. Transcatheter occlusion of the persistently patent ductus arteriosus. Forty-month follow-up and prevalence of residual shunting. Circulation 1991;84:2313-7.

4 Musewe NN, Benson LN, Smallhorn JF, Freedom RM. Two-dimensional echocardiographic and colour flow Rashkind prosthesis. Circulation 1989;80:1706-10.

5 Dyck JD, Benson LN, Smallhorn JF, McLaughlin PR, Freedom RM, Rowe RD. Catheter occlusion of the persistently patent ductus arteriosus. Am $f$ Cardiol sistently patent

6 Rohmer J, Hess J, Talsma MD. Closure of the persistent ductus arteriosus (Botalli) using a catheter procedure; the initial 50 patients treated in the Netherlands [English abstract]. Ned Tijdschr Geneeskd 1990;134: 2347-51.

7 Hosking MCK, Benson LN, Freedom RM. Reocclusion for persistent shunting after catheter placement of the patent ductus arteriosus occluder. Can F Cardiol 1989, 5:340-2. 
8 Ladusans EJ, Murdoch I, Franciosi J. Severe haemolysis after percutaneous closure of a ductus arteriosus (arterial duct). Br Heart $₹$ 1989;61:548-50.

9 Benson LN, Dyke JD, Hecht B. Catheter closure of the small patent ductus arteriosus. Cathet Cardiovasc Diagn small patent

10 Gardner MJ, Altman DG. Statistics with Confidence. London: British Medical Journal Publications. 1989: London:

11 Campbell $M$. Natural history of persistent ductus arteriosus. $\mathrm{Br}$ Heart $\mathcal{F}$ 1968;30:4-13.

12 Gross RE. The patent ductus arteriosus. Observations on diagnosis and therapy in 525 treated cases. Am $\mathcal{F}$ Med 1952;12:472-82.

13 Kirklin JW, Barratt-Boyes BG. Cardiac Surgery. New York: John Wiley, 1986:684-92.

14 Sørensen KE, Kristensen BØ, Hansen OK. Frequency of occurrence of residual ductal flow after surgical ligation by color-flow mapping. Am 7 Cardiol 1991;67:653-4.

15 Hayes AM, Redington AN, Rigby ML. Severe haemolysis after transcatheter duct occlusio

dy. Br Heart f 1992;67:321-2. Houston AB, Gnanapragasam JP, Lim MK, Doig WB,
Coleman EN. Doppler ultrasound and the silent ductus Coleman EN. Doppler ultrasound and

17 Latson LA. Residual shunts after transcatheter closure of patent ductus arteriosus; a major concern or benign "techno-malady". Circulation 1991;6:2591-3.

18 Bayliss R, Clarke C, Oakley C, Somerville W, Whitfield AGW. The teeth and infective endocarditis. Br Heart $\mathcal{f} \frac{T}{\mathbb{D}}$ 1983;50:506-12.

19 Van Der Meer JT, Van Wijk W, Thompson J, Vandenbroucke JP, Valkenburg HA, Michel MF. $\subseteq$ Efficacy of antibiotic prophylaxis for prevention of
native-valve endocarditis. Lancet 1992;339:135-9.

\section{ABSTRACTS IN CARDIOLOGY}

\section{Of claret and coronaries}

Despite enjoying a cuisine rich in saturated fats, the French escape the high incidence of coronary artery disease to which their neighbours across the Channel are subject. The reason for this protection has been attributed, in part, to the moderate and regular consumption of wine-specifically red wine.

Frankel et al investigated the hypothesis that the antioxidant properties of the complex phenolic substances found in red wine (flavonols, catechins, anthocyanins, and soluble tannins) may protect low density lipoprotein (LDL) from peroxidation to an atherogenic form. They isolated wine phenolics from a Californian red of the Petite Sirrah grape variety. (Why French wine was not used is not explained-perhaps they preferred to drink it?) They showed that 1000 fold dilutions of red wine phenolics were effective in inhibiting the copper catalysed oxidation of LDL.

This in vitro observation cannot be extrapolated to explain the rates of coronary artery disease in France. Copper catalysed oxidation $\triangle$ of LDL does not occur in vivo, and little is $\overrightarrow{\vec{O}}$ known of the absorption and pharmaco- 3 kinetics of these phenolic compounds, or of the biological effects of LDL exposed to red wine derivatives. So we are left to mull over $\vec{\sigma}$ the effects the next glass of red wine may be $\frac{\mathrm{m}}{2}$ having on our coronary arteries. Santé!

\section{Inhibition of oxidation of human low-density lipoprotein by phenolic substances in red wine}

E N Frankel, I Karnior, I B Ceman, Es Porks, I E Kinsello

Abotinater

The "French paradox" (apparent compatibithy of a high fat diet Whin a low incidence of coromaty athercosclerosis) iths been atribured to the fegular drinking of red whe. Itowever, the alcohol content of wine tant not be the sole eroplination for this protection. Ret yine also conteins bhenolic compounds, snd the antioridht poopertes of these mat hore an twitortin role.

In in viro stutiles with phenolic subatances in red wine and normal human low-density lipoprotein (MDL) we found that red wine inhibits the copper-catalysed ofidmion of LDL. Wine diluted 1000 -rold containing 10 minilh total phenolics inhibitod IDI oxidation significantly more than atoconherol.

Oin tindings show that the non-alcoholic components of red wive have potent antioxidant propertes toward oxtiation of human LDL. (Lancet $1993,34(1 \times 454-7)$ 